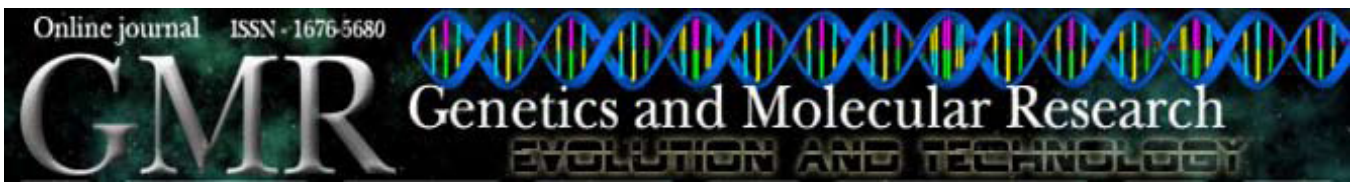

\title{
Cytogenetic evidence for de novo synthesis of rRNA and involvement of nucleolar material in the organization of cell structures during spermiogenesis of Chariesterus armatus (Heteroptera, Coreidae)
} \author{
M.M. Itoyama \\ Laboratório de Citogenética e Molecular de Insetos, \\ Departamento de Biologia, Instituto de Biociências, \\ Letras e Ciências Exatas, Universidade Estadual Paulista, \\ São José do Rio Preto, SP, Brasil \\ Corresponding author: M.M. Itoyama \\ E-mail: mary@ibilce.unesp.br
}

R.L.M. Arakaki, H.V. Souza, M.M.U. Castanhole, H.E.M.C. Bicudo and

Genet. Mol. Res. 9 (3): 1877-1885 (2010)

Received May 21, 2010

Accepted July 15, 2010

Published September 21, 2010

DOI 10.4238/vol9-3gmr930

\begin{abstract}
The nucleolar material of Chariesterus armatus was analyzed during spermiogenesis in cell preparations impregnated with silver nitrate. Nucleolar corpuscles were observed in spermatids at the beginning of the process, showing that this organoid is also maintained after meiosis. In addition, nucleoli were seen in the round spermatids connected to the X-chromosome (bearer of the nucleolar organizer in C. armatus), indicating de novo synthesis of nucleolar material. This differs from the reorganization of ribosomal granules, transported from meiotic spermatocytes to round spermatids, where they would support protein synthesis, which is reported for other species. We also observed connections of nucleolar corpuscles to the nuclear membrane regions where the tail and the acrosome will be formed, suggesting
\end{abstract}


close involvement of the nucleolar material in the formation of these structures. In addition to the nucleolar bodies, we detected silver-positive structures, which will require new approaches to clarify their role. One of these structures, observed in the cytoplasm, appears to correspond to the chromatoid body, which has been found in several organisms, but is still poorly understood; another is a complex structure to which the tail appears to be connected. We conclude that $C$. armatus is an appropriate model for understanding not only the synthesis of rRNA in the spermiogenesis, but also the functional meaning of the close relationship of nucleolar material with other structures during this process.

Key words: Spermiogenesis; Silver impregnation; Chromatoid body; Nucleolar synthesis in spermatid; Nucleolus-acrosome;

Nucleolus-tail relations

\section{INTRODUCTION}

The Brazilian species of Heteroptera (Coreidae family) Chariesterus armatus is economically important because it is a plantation pest. Testicular cells of this species were previously analyzed regarding cytogenetic characteristics showing that its karyotype is formed by $2 \mathrm{n}=25$ (Souza et al., 2007).

In general, cytogenetical information on the spermiogenesis of Heteroptera (mainly in the Coreidae family) is missing in the literature and this also includes the behavior of the nucleolar material during the process. It is notorious that the nucleolar material plays an important role in protein synthesis and other aspects of cell life (Lewin, 2007). Since the reproductive cells are subjected to a complex differentiation in order to become spermatozoa they must make intensive use of synthesis in the sequence of transformations.

Studies of the nucleolar behavior in meiosis and spermiogenesis of different organisms have shown some peculiarities. One of them is named nucleolar persistence (Simmonds and Dodds, 1947; Nankin et al., 1970). This phenomenon differs from the more general process in which the disorganization of the nucleolus occurs during prophase in that, from diakinesis until the end of the meiotic division, it is not visible, suggesting that at the time it is already completely disorganized (Risueño and Medina, 1976). Nucleolar persistence, that is, the maintenance of visible nucleolar material after meiotic prophase, has been described in several Heteroptera species (Morielle-Souza and Azeredo-Oliveira, 2007; Costa et al., 2008; Souza et al., 2008). In Mormidea v-luteum, Oebalus poecilus and Oebalus ypsilongriseus (three Heteroptera species from the Pentatomidae family) the nucleolar material could be observed as a round corpuscle until the end of telophase I, and the round spermatids still exhibited a variable number of small nucleoli. When the spermatid started to elongate, the silver nitrate-stained material concentrated at the posterior region of the head, where it was seen until completing the formation of the spermatozoa (Souza et al., 2008). Nucleolar persistence in human spermatid was also detected by fluorescence in situ hybridization (FISH) (Dadoune et al., 1994).

Another aspect of the nucleolar behavior in spermatogenesis is that, over time, several observations have suggested that the nucleolar granules or masses, which persist 
at the end of meiosis, reorganize the nucleolus in early spermiogenesis and support protein synthesis in the process (Chathoth et al., 2009). The idea that nucleolar synthesis does not occur in spermiogenesis has been supported by the finding of a strong down regulation of nucleolin, a major nucleolar component in eukaryotic cells that plays a significant role in ribosomal RNA transcription (Richards et al., 2007). However, the identification of new variants of nucleolin, which have been recently reported in Drosophila and human spermatogenesis, hypothetically has the potential to support rDNA synthesis in the haploid cells (Mikhaylova et al., 2006).

In the present study, the testicular cells of $C$. armatus were analyzed as to the morphological modifications of the nucleolar material during spermiogenesis. The observations suggested de novo nucleolar synthesis in spermatids and interactions of the nucleolar material with other cell structures during the process that, if adequately approached, may reveal important details on the spermatozoon differentiation process.

\section{MATERIAL AND METHODS}

Ten adult $C$. armatus males were analyzed. They were collected in São José do Rio Preto (2047'32” S, 49²1'37' W), SP, Brazil, from Gossypium hirsutum L. (cotton) plants.

Preparations were made as follows: the testes were dissected in Demerec solution (Demerec and Kaufmann, 1945), squashed in 50\% aqueous acetic acid solution and impregnated with silver nitrate according to the protocol described by Howell and Black (1980). This technique has been intensively used as a marker for nucleolar organizing regions (NORs) and nucleoli. Proteins necessary for rRNA transcription and pre-ribosome processing are able to reduce silver nitrate, acquiring a dark brown color (Schwarzacher and Wachtler, 1993). The validity of this procedure is reinforced by studies in which other specific techniques have been used marking the same regions [acridine orange (de Oliveira and Campos Bicudo, 2006); FISH (Purwantoro and Takato, 1998; Margarido and Galetti Jr., 2000)]. Bernard et al. (2001) successfully applied silver staining to eliminate false-negative results from whole mount immunocytochemistry due to insufficient individual antigen concentration.

Prints for preparing the figures were obtained using a Zeiss microscope (AXIO VISION program) owned by the Morphology Laboratory of the Department of Biology in IBILCE, UNESP, São José do Rio Preto, SP, Brazil.

\section{RESULTS}

Observations of the nucleolar material during the spermiogenesis of C. armatus are shown in Figure 1. A nucleolar corpuscle surrounded by a fine "ring" with a prolongation that gives it a racket shape was initially visible at the center of the early round spermatid nuclei while a small elevated "vacuole" was observed in the nuclear membrane on the opposite side of the racket handle-like structure (Figure 1a). Later in the process, the small nucleolar corpuscle could no longer be seen, but the vacuole remained.

At this time, a formation differing from the surrounding material with a denser structure was visible in the cytoplasm, in contact with the external part of a nucleus membrane region. Inside this formation there was an intensely silver-stained corpuscle connected with 


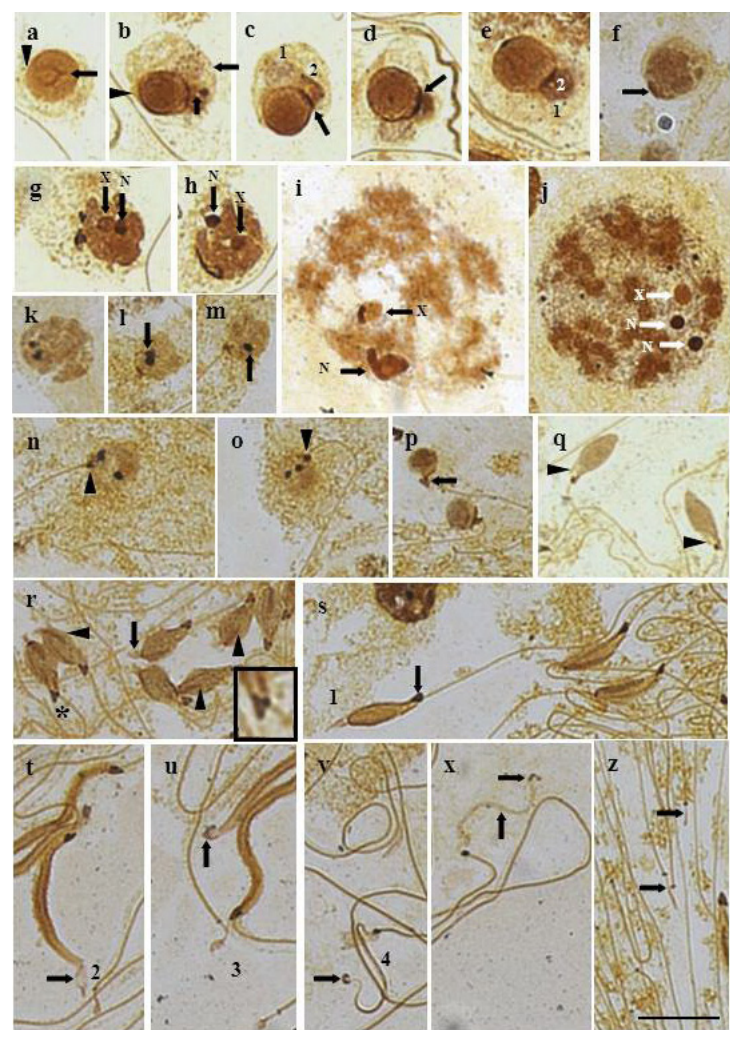

Figure 1. Behavior of silver-positive structures in the spermiogenesis of Chariesterus armatus (Coreidae, Heteroptera). (a) Presence of a small central silver-positive corpuscle in the nucleus of the round spermatid, surrounded by a racketshaped ring (arrow), and a vesicular salience (arrowhead) in the nuclear membrane region on the opposite side of the "racket handle". (b) In the cytoplasm, presence of a differentiated structure that contacts the nuclear membrane (long arrow), inside of which there is an intensely silver-stained corpuscle associated with the nucleus membrane by a filament (short arrow); the vesicular formation is maintained on the opposite side of the nuclear membrane (arrowhead). (c-e) The cytoplasmatic structure appears to be formed by two regions (1 and 2) of different densities and degrees of staining, when posed side by side; the cytoplasmatic silver-positive granules disappear; the region of the nuclear membrane in contact with the more intensely stained portion of the cytoplasmatic structure presents an intensely stained thickening (arrows); in $e$, the most dense part in the structure appears to be included in the less dense one ( 1 and 2$)$. (f) The cytoplasmatic structure disappears, but the nuclear membrane region remains thick (arrow); the chromatin begins a condensation process. $(\mathrm{g}, \mathrm{h}$ ) The X-chromosome is differentiated amid the chromatin agglomerates, and a nucleolar corpuscle $(\mathrm{N})$ is seen associated with the X-chromosome (X). ( $\mathrm{i}, \mathrm{j})$ Two prophase cells that confirm the involvement of the X-chromosome in the nucleolar synthesis, indicating that the NOR is located on the X-chromosome. (k) Two nucleolar corpuscles are seen in this cell. (1-o) The chromatin condenses again and two or three nucleolar corpuscles are observed in the cells (arrows). (m-o) In this phase, in which the nucleus is still round, there is an association by filament of a nucleolar corpuscle with the also silver-positive place where the tail is inserted (arrowheads). (n-p) Nucleolar corpuscles are no longer visible. (q) Elongated nucleus: a "vacuole" can be observed on the side of the tail insertion region (arrowheads). (r, s) From this phase on, there is an alteration in the structure to which the tail associates (the inset was prepared from the region marked with an asterisk); it presents two joined "plates" or "disks", of different sizes and different staining intensities, with the tail associating apparently only with the "smaller disk"; filaments run lengthwise through the spermatid head and a fine line departs from the acrosomal region to the interior of the nucleus (arrowheads); there is also the beginning of a long acrosome formation (arrow). (s-v) Sequential aspects (1 to 4 ) in the formation of the acrosome, exhibiting a silver-positive tip (arrow in $\mathrm{u}$ and $\mathrm{v}$ ); from $r$ the elongation of a fine line in the direction of the tail insertion extremity is observed. (v-z) The acrosome remains long even when the spermatozoon head is already an intensely silver-stained dot (arrows). Bar: $10 \mu \mathrm{m}$. 
the nucleus membrane by a filament (Figure 1b). As the process proceeded, this granule disappeared and the nuclear membrane became thicker and intensely stained in the region to which it had been connected (Figure 1c).

The cytoplasmatic formation associated with the nucleus membrane was formed by two parts of different densities and degrees of coloration. The nuclear membrane in the region in contact with the more intensely stained part was thicker than the membrane region in contact with the less intensely stained one (Figure 1c,d). Next, the less intensely stained part was apparently included in the more intensely stained one (Figure 1e). This structure, located in the cytoplasm but connected to the nucleus, disappeared as the spermiogenesis process continued, but the contact region in the nuclear membrane remained thick (Figure 1f).

The chromatin, which appeared almost homogeneous until that time, now formed agglomerates (Figure 1g,h). Inside the nucleus, amid these chromatin agglomerates, the Xchromosome could be observed with a more homogeneous structure and one nucleolar corpuscle associated with it (Figure 1g,h). To reinforce this observation, two prints of prophase cells, indicating that in C. armatus the NOR is located on the X-chromosome, were included in Figure 1 (Figure 1i,j); in Figure 1j, two nucleolar bodies are present.

Generally, two nucleolar corpuscles separated from the X-chromosome were seen at this stage of spermiogenesis, when the chromatin had already recovered a homogeneous appearance (Figure 1k-o). One of them now appeared connected by a thin filament to the more intensely stained region of the nuclear membrane to which the tail was already associated (Figure $1 \mathrm{~m}-\mathrm{o}$ ). Afterwards, only the region of the tail insertion remained intensely stained (Figure 1p).

At the beginning of the spermatid elongation, the silver staining was restricted to the corpuscle associated with the tail. A kind of elongated "vacuole" was observed on one of the sides on the extremity where the tail was inserted (Figure 1q).

As the process continued, the anterior part of the head (acrosome region) also showed silver staining; from it, a lateral band led to the interior of the nucleus, about one third or half of its length. A filament could also be seen sidelong, accompanying the head length. At this time, the long protrusion of the acrosome was already starting to form (Figure 1r) and the structure present in the insertion place of the flagellum was more complex (Figure 1r-t).

The spermatid elongation intensified and the lateral filament elongated more towards the tail insertion region (Figure 1s,u). The acrosome structure became longer (numbers 1 to 4 , in Figure 1s-v) and, in its distal extremity, emerged a silver-positive "cap" that resembled an arrow tip, and might be formed by the membrane region previously stained with silver ions that was "pushed" by the acrosome growth (Figure 1v,x).

The structure present in the region where the flagellum was inserted seemed to be composed of two joined plates of different lengths, the smaller centralized in relation to the larger. The larger one stained less intensely than the smaller one (Figure 1r and inset). Apparently, the tail was associated only with the smaller, intensely stained plate (Figure 1r-u).

At the end of the spermiogenesis, the small round spermatozoon head showed an intense impregnation with the silver ions and the acrosome, partially shortened, could still be observed in some of them (Figure 1x,z).

\section{DISCUSSION}

The protocols for silver ion impregnation, described by Goodpasture and Bloom (1975) 
and Howell and Black (1980), have been applied to specifically highlight the nucleolar material and the chromosomal NORs in many organisms. Proteins necessary for rRNA transcription and pre-ribosome processing are able to reduce silver nitrate, acquiring a dark brown color. Thanks to these techniques, the knowledge about nucleolar activity and its regulation has advanced greatly in a relatively short time (Bicudo, 1982; Capoa et al, 1985; Shaw and Jordan, 1995; Santoro et al., 2002; de Oliveira and de Campos Bicudo, 2006; Severi-Aguiar et al., 2006; Das and Khuda-Bukhsh, 2007; Castanhole et al., 2008). In the present study, the protocol of Howell and Black (1980) was used, yielding information on morphological changes and interactions of the nucleolar material during the spermiogenesis of the heteropteran C. armatus.

One of the first observations to be highlighted in this study is the presence of nucleolar corpuscles in the round spermatid. They appeared silver stained in the nucleus right at the beginning of spermiogenesis. The presence of nucleolus in the spermatids of some organisms has been known for a long time. Sotelo and Trujillo (1954) are among the first authors to devote attention to demonstrate this fact, using arachnids as a model. Those authors stated that, "the whole nucleolus (nucleolonema and amorphous pairs) is evidently a permanent structure because it was found in the fertilizing spermatozoon and consequently has to be in the future masculine pronucleus". In C. armatus, we observed the presence of a nucleolus in the early round spermatid, but a little later, still in the round stage, the chromatin condensed and one or two nucleolar corpuscles appeared associated with the X-chromosome, which contains the NOR in this species. This observation indicated de novo synthesis of the nucleolar material. Therefore, the constant presence of the nucleolus during spermiogenesis may involve not only a reorganization of dispersed granules, as previously thought, but also a new synthesis.

Another interesting observation was the early presence of a morphological differentiation in the regions of the nuclear membrane where the tail would be inserted and the acrosome would develop, suggesting that the position of these structures is predetermined, an interesting problem that deserves further research. In addition, associations of the nucleolar corpuscles with those regions, still in the round spermatid phase, may suggest an apparently direct participation of the nucleolar material in the process of their formation. How this occurs remains to be understood. In relation to the acrosome, it is easy to suppose an intense activity of synthesis for the production of enzymes to be stored in that structure, provided that its primary function is the rupture of the ovum membrane.

In general, the observations in this study indicated that there is high metabolic activity involving the nucleolar material during spermiogenesis. This idea finds support from Monesi (1965) to whom the transcription of mRNA ceases in initial spermatids, when its nucleus begins to elongate, but the protein synthesis continues until the final stages of spermiogenesis. This statement leads to the conclusion that the presence of ribosomes, the basic component of the nucleolus that "reads" the mRNA sequence for producing protein, is necessary during the whole spermiogenesis process.

Another structure intensely stained by silver ions and observed early in the spermiogenesis of this species was a corpuscle, present not in the nucleus but in the cytoplasm, which was connected to the nuclear membrane by a thin filament. Data on other organisms suggested that it may correspond to the chromatoid body (CB) described by Sud (1961), and other authors such as Söderström and Parvinen (1976). In addition to the morphological similarity, the information that in the crayfish Cambarus virilis CB stains intensively with silver ions (McCroan, 1940, in Sud, 1961) reinforces the idea of this correspondence. According to Sud 
(1961), CB consists mainly of RNA and proteins, suggesting that this structure may be a center of protein synthesis. Kotaja et al. (2006) defined CB as a "perinuclear, cytoplasmic cloud-like structure in male germ cells" that in mice can act like a storage and processing location for the RNA, occupying a privileged position in the post-transcriptional control of gene expression. The connection of this structure with the nucleus, observed in the present study, suggests a nucleus-cytoplasmatic interaction.

CBs have already been described in many organisms and were predominantly analyzed in rodents (Parvinen, 2005). Data provided by Alvestad-Graebner and Adam (1976) support the idea that CB plays a role in the development of acrosomes in young spermatids. The same is suggested by Söderström and Parvinen, (1976), Tang et al. (1982) and Anton (1983). Although the CB was discovered in the 19th century (von Brunn, 1876, in Sud, 1961), its origin, behavior and function are still poorly understood, as shown by the great diversity and even conflicting information present in literature. In the present study, the observations support the involvement of the supposed CB in the differentiation of the tail.

Another controversial aspect about the $\mathrm{CB}$ refers to its origin. According to some authors, its origin is nuclear, but other observations support a cytoplasmatic origin (AlvestadGraebner and Adam, 1976). In the present study, the intense silver impregnation of the supposed $\mathrm{CB}$ using a technique specific for nucleolar material reinforces the nuclear origin.

The morphology of the CB shows a great variation among different organisms. The only paper we could find in the literature related to CB in Heteroptera was the one by Wilson (1913, in Sud, 1961). In that study, performed with Pentatoma senilis (Hemiptera,) CB was described as an apparent central cavity with an occasional central granule intensely stained. In the present study, the intensively stained corpuscle appears inside a complex structure that changes morphologically before its disappearance in the round spermatid phase.

Among the observations in the present study, we also highlight the silver-stained structure to which the tail is associated, apparently formed by two joined "plates" of different sizes and different degrees of silver impregnation. It seems that the tail is connected only to the smaller and more intensely stained plate. The role of the very long acrosome is also an interesting question.

Additional approaches using this species may explore more deeply the functional meaning of the present observations on important and still poorly understood aspects of the spermiogenesis process.

\section{ACKNOWLEDGMENTS}

Special thanks to Sônia Maria Oliani of the Department of Biology of the IBILCE/ UNESP for assistance with the cell images, and FUNDUNESP and FAPESP for financial support.

\section{REFERENCES}

Alvestad-Graebner I and Adam H (1976). Relationship between the chromatoid body and the acrosomal system in early spermatids of Myxine glutinosa L. Cell Tissue Res. 174: 427-430.

Anton E (1983). Association of Golgi vesicles containing acid phosphatase with the chromatoid body of rat spermatids. Experientia 39: 393-394.

Bernard J, Canet V, Giroud F, Montmasson MP, et al. (2001). Quantitation of AgNORs by flow versus image cytometry. 
J. Histochem. Cytochem. 49: 433-437.

Bicudo HEMC (1982). Silver staining and the nucleolar organizing activity in Drosophila species of the mulleri complex and their hybrids. Rev. Bras. Genet. 1: 31-50.

Capoa de A, Marlekaj P, Baldini A, Rocchi M, et al. (1985). Cytologic demonstration of different activity of rRNA gene clusters in different human tissues. Hum. Genet. 69: 212-217.

Castanhole MM, Pereira LL, Souza HV, Bicudo HE, et al. (2008). Heteropicnotic chromatin and nucleolar activity in meiosis and spermiogenesis of Limnogonus aduncus (Heteroptera, Gerridae): a stained nucleolar organizing region that can serve as a model for studying chromosome behavior. Genet. Mol. Res. 7: 1398-1407.

Chathoth KT, Ganesan G and Rao MRS (2009). Identification of a novel nucleolin related protein (NRP) gene expressed during rat spermatogenesis. BMC Mol. Biol. 10: 64 .

Costa AC, Azeredo-Oliveira MTV and Tartarotti E (2008). Spermatogenesis and nucleolar activity in Triatoma klugi (Triatomine, Heteroptera). Genet. Mol. Biol. 31: 438-444.

Dadoune JP, Siffroi JP and Alfonsi MF (1994). Ultrastructural localization of rDNA and rRNA by in situ hybridization in the nucleolus of human spermatids. Cell Tissue Res. 278: 611-616.

Das JK and Khuda-Bukhsh AR (2007). Preponderance of GC-rich sites in silver-stained nucleolus organizing regions of Rita rita (Hamilton) and Mystus gulio (Hamilton) (Bagridae, Pisces), as revealed by chromomycin A3-staining technique and scanning electron microscopic studies. Genet. Mol. Res. 6: 284-291.

de Oliveira CC and de Campos Bicudo HE (2006). A new nucleolar body appears in Drosophila saltans salivary gland cells before histolysis, in programmed cell death. J. Insect Sci. 6: 1-7.

Demerec M and Kaufmann BP (1945). Drosophila Guide. The Lord Baltimore Press, Baltimore.

Goodpasture C and Bloom SE (1975). Visualization of nucleolar organizer regions in mammalian chromosomes using silver staining. Chromosoma 53: 37-50.

Howell WM and Black DA (1980). Controlled silver-staining of nucleolus organizer regions with a protective colloidal developer: a 1-step method. Experientia 36: 1014-1015.

Kotaja N, Bhattacharyya SN, Jaskiewicz L, Kimmins S, et al. (2006). The chromatoid body of male germ cells: similarity with processing bodies and presence of Dicer and microRNA pathway components. Proc. Natl. Acad. Sci. U. S. A. 103: 2647-2652.

Lewin B (2007). Protein Synthesis. In: Genes IX (Lewin B, ed.). Jones \& Bartlett Publishers, Inc., Massachusetts, 151-183.

Margarido VP and Galetti PM Jr (2000). Amplification of a GC-rich heterochromatin in the freshwater fish Leporinus desmotes (Characiformes, Anostomidae). Genet. Mol. Biol. 23: 569-573

Mikhaylova LM, Boutanaev AM and Nurminsky DI (2006). Transcriptional regulation by modulo integrates meiosis and spermatid differentiation in male germ line. Proc. Natl. Acad. Sci. U. S. A. 103: 11975-11980.

Monesi V (1965). Synthetic activities during spermatogenesis in the mouse RNA and protein. Exp. Cell Res. 39: 197-224.

Morielle-Souza A and Azeredo-Oliveira MTV (2007). Differential characterization of holocentric chromosomes in triatomines (Heteroptera, Triatominae) using different staining techniques and fluorescent in situ hybridization. Genet. Mol. Res. 6: 713-720.

Nankin HR, Brecher S and Mullins J (1970). In vitro alteration of satellite association and nucleolar persistence in mitotic human lymphocytes. Cytogenet. Genome Res. 9: 42-51.

Parvinen M (2005). The chromatoid body in spermatogenesis. Int. J. Androl. 28: 189-201.

Purwantoro A and Takato K (1998). Localization of rRNA genes in Lilium by fluorescence in situ hybridization and its comparison with silver staining. Chromosome Sci. 2: 77-82.

Rickards B, Flint SJ, Cole MD and LeRoy G (2007). Nucleolin is required for RNA polymerase I transcription in vivo. Mol. Cell Biol. 27: 937-948.

Risueño MC and Medina FJ (1976). The nucleolar structure in plant cells. Rev. Biol. Cel. 7: 1-162.

Santoro R, Li J and Grummt I (2002). The nucleolar remodeling complex NoRC mediates heterochromatin formation and silencing of ribosomal gene transcription. Nat. Genet. 32: 393-396.

Schwarzacher HG and Wachtler F (1993). The nucleolus. Anat. Embryol. 188: 515-536.

Severi-Aguiar GD, Lourenco LB, Bicudo HE and Azeredo-Oliveira MT (2006). Meiosis aspects and nucleolar activity in Triatoma vitticeps (Triatominae, Heteroptera). Genetica 126: 141-151.

Shaw PJ and Jordan EG (1995). The nucleolus. Annu. Rev. Cell Dev. Biol. 11: 93-121.

Simmonds NW and Dodds KS (1947). The persistence of a nucleolar remnant during meiosis in a diploid Banana. Ann. Bot. 11: 369-374.

Söderström KO and Parvinen M (1976). Transport of material between the nucleus, the chromatoid body and the Golgi complex in the early spermatids of the rat. Cell Tissue Res. 168: 335-342.

Sotelo JR and Trujillo O (1954). On the presence and character of the nucleolus in the spermatid and fecundant spermatozoons of Heteropachyloidellus robustus Roew. Z. Zellforsch. Mikrosk. Anat. 39: 470-478.

Genetics and Molecular Research 9 (3): 1877-1885 (2010)

CFUNPEC-RP www.funpecrp.com.br 
Souza HV, Arakaki RLM, Dias LN, Murakami AS, et al. (2007). Cytogenetical aspects of testicular cells in economically important species of Coreidae family (Heteroptera). Cytologia 72: 49-56.

Souza HV, Castanhole MMU, Bicudo HEMC, Costa LAA, et al. (2008). Morphological patterns of the heteropycnotic chromatin and nucleolar material in meiosis and spermiogenesis of some Pentatomidae (Heteroptera). Genet. Mol. Biol. 31: 689-691.

Sud BN (1961). The "chromatoid body" in spermatogenesis. Science 102: 273-292.

Tang XM, Lalli MF and Clermont Y (1982). A cytochemical study of the Golgi apparatus of the spermatid during spermiogenesis in the rat. Am. J. Anat. 163: 283-294. 